\title{
The General Assessment of Problem Solving Processes and Metacognition in Physics Education
}

\author{
Tolga Gok ${ }^{*}$ \\ University of Dokuz Eylul, Torbali Vocational School of Higher Education, Izmir, Turkey
}

Received 5 July 2010 - Accepted 23 August 2010

\begin{abstract}
Problem solving is one of the primary tools for college and university science instruction. In this study, the review of problem solving and metacognition skills of students was presented. Basically, at the first step, problem solving was defined and then the differences of the experienced and inexperienced problem solvers were considered. Various strategy steps of problem solving reported in the open literature were discussed. Metacognition was introduced as an important part of problem solving process. The research available in the literature indicated that teaching problem solving strategies help students but not sufficient to promote true science expertise. Meta-cognitive skills should be clearly taught to build structured knowledge and develop desirable habits of mind, and to guide students through the stages of cognitive development.
\end{abstract}

Key words: Metacognition, Problem Solving, Physics Education, Physics Problems

\section{Introduction}

\section{The Definition of Problem Solving}

Problem solving has been acknowledged as a paradigm of complex cognition that is part of our everyday experience. Most researchers working on problem solving (Dewey, 1910; Newell \& Simon, 1972; Elshout, 1987; Mayer, 1991; Schunk, 2000) agree that a problem occurs only when someone is confronted with a difficulty for which an immediate answer is not available. However, difficulty is not an intrinsic characteristic of a problem because it depends on the solver's knowledge and experience (Garrett, 1986; Gil-Perez et al., 1990). So, a problem might be a genuine problem for one individual but might not be for another (Schunk, 2000).

Problem solving is viewed as a fundamental part of learning science in regular schools (Reif et al., 1976; Larkin \& Reif, 1979; Chi et al., 1981; Reif, 1981; Bascones et al., 1985; Amigues, 1988; Robertson, 1990; Savage \& Williams, 1990; McDermott, 1991; Heller et al., 1992; Henderson et al., 2001; Kuo, 2004; Pol, 2005; Yerushalmi \& Magen, 2006; Loucks, 2007). After the instructor introduces the concepts, students apply these concepts in problems. Problems in this context follow some well-defined criteria: all information needed to solve the problem is given; a limited set of rules is needed to solve the problem; in many cases only one procedure leads to the right answer; and there is only one correct answer. This kind of

* Corresponding author: Phone: +90 23285318 20,

Email: tolga.gok@deu.edu.tr

ISSN: 1306-3049, (C)2010 
problem is rather common in educational practice, but problem solving in this approach is “dominated by recall, a relatively undemanding cognitive task” (Osborne \& Dillon, 2008).

Problem solving as a goal-directed behavior requires an appropriate mental representation of the problem and the subsequent application of certain methods or strategies in order to move from an initial, current state to a desired goal state (Metallidou, 2009).

\section{The Difference of the Expert and Novice in Problem Solving}

Research on developing an effective instruction for physics problem solving started at least 50 years ago (Garrett, 1986) and changed after the late 1970s (Larkin \& Reif, 1979; Larkin et al., 1980; Chi et al., 1981; Heller \& Reif, 1984; Reif, 1995; Dufrense et al., 1997; Kohl et al., 2007; Kohl \& Finkelstein, 2008).

Most of the research during this period aimed to identify the differences between experienced and inexperienced problem solvers. Larkin (1979) showed inexperienced problem solvers tend to spend little time representing the problem and quickly jump into quantitative expressions. Also, they perform problem solving techniques that include haphazard formula-seeking and solution pattern matching (Reif et al., 1976; Van Heuvelen, 1991; Mazur, 1997). By contrast, experienced problem solvers solve problems by interjecting another step of a qualitative analysis or a low-detail review of the problem before writing down equations. This qualitative analysis, such as a verbal description or a picture, serves as a decision guide for planning and evaluating the solution (Larkin \& Reif, 1979). Although this step takes extra time to complete, it facilitates the efficient completion of further solution steps and usually the experienced problem solver is able to successfully complete the problem in less time.

Reif and Heller (1982) discussed how two solvers organize and use their knowledge after reading the problem. Inexperienced solvers do not necessarily have the knowledge structure, as their understanding consists of random facts and equations that have little conceptual meaning and they access each principle or equation individually from memory. Larkin (1979) suggested that experienced solvers store physics principles in memory as chunks of information that are connected and can be usefully applied together. Experienced solvers have lower cognitive load on their short-term memory and can devote more memory to the process of solving the problem (Sweller, 1988). They also approach the tasks differently from those with less practice and classify problems qualitatively and according to major principles (Mestre, 2001).

Kohl and Finkelstein (2008) concluded that experienced solvers are more successful while solving problems that required the use of multiple representations, finish faster, and move more quickly among the representations available. Other solvers are just as likely to use multiple representations extensively in their solutions and that they use very similar selections of representations. This may be because doing so has become the norm in these representation rich physics classes, and so they draw pictures and free-body diagrams regardless of their understanding of why.

However, Schoenfeld (1985) claimed that in order to solve mathematics problems, both experienced and inexperienced solvers use the same episodes of orientation, analysis, planmaking, implementation, and verification. The only difference is experienced solvers take relatively more time to read and analyze problems and to "look back", whereas inexperienced solvers devote most time to finding a solution plan and calculating. According to them this is because of teaching system which does not cover how to do so and does not possess general problem solving strategies to manage their own problem solving actions. 
It is for sure that it's not just the way physicists read and use the equations that are different from math. Also the goals are different. They don't just want to explore ways of solving equations, they want to describe, learn about, and understand physical systems. However, the step should be followed to reach the target, in this case solution, will be similar. Based on the findings in the literature of different disciplines, researchers started to examine novice students' difficulties in confronting real problems and how they can overcome these difficulties. They investigated the methods used by experienced solvers and tried to define some problem solving patterns, a general instruction guideline which can meet the various patterns of problem solving.

\section{Problem Solving Strategies in Problem Solving}

Most of the researchers examined on general and specific problem solving strategies. The most notably general strategies are Polya's (1945), Dewey’s (1910), and Kneeland (1999) problem solving strategy steps.

Dewey (1910) cited for his four steps (problem's location and definition, suggestion of possible solution, development by reasoning the bearings of the solution, and further observation and experiment leadings to its acceptance or rejection) of problem solving strategy.

Polya (1945) cited for his four steps problem solving strategy as follows: First step is Understanding the Problem, by identifying the unknown, the data, and the condition, and then drawing a figure and introducing suitable notation. The second step is Devising a Plan, in which the solver seeks a connection between the data and the unknown. If an immediate connection is not found, the solver considers related problems or problems that have already been solved, and uses this information to devise a plan to reach the unknown. In the third step, Carrying out the Plan, the steps outlined in part two are carried out, and each step is checked for correctness. In the final step Looking Back, the problem solution is examined, and arguments are checked.

Reif et al. (1976) tried to teach students a simple problem solving strategy consisting of the following four major steps: Description, which lists clearly the given and wanted information. Draw a diagram of the situation. The next step, Planning, selects the basic relations suitable for solving the problem and outline how they are to be used. The step Implementation performs the preceding plan by doing all necessary calculations. The final step is Checking, which checks that each of the preceding steps was valid and that the final answer makes sense.

Reif (1995) improved early problem solving strategies in his textbook "Understanding Basic Mechanics”. Revised steps included in the book were: Analyze the Problem, in which a basic description of the situation and goals is generated, and a refined physics description according to time sequences and intervals is developed. The second step is Construction of a Solution, in which basic useful relations are identified and performed until unwanted quantities are eliminated. The final step is called Check, and asks the solver if the goal has been attained, the answer is with known quantities, and there is consistency within the solution with units, signs, and sensibility of values.

Kneeland's (1999) problem solving model contains of six separate steps: awareness of the problem, gathering of relevant facts, definition of the problem, development of solution options, selection of the best solution, and implementation of the solution. The first three steps have to do with defining the problem. The next three steps move students from the understanding phase into the solution phase: exploring and developing various solution options and then acting on the best one. 
Over the past 40 years, in addition to these strategies, numerous physics problem solving methods have been published to help students improve their understanding and problem solving. These are the logical problem solving model; the didactic approach; the collaboration method (Harskamp \& Ding, 2006); the computer-assisted instruction model (Bolton \& Ross, 1997; Pol, 2005); the translating context-rich problem approach (Heller et al., 1992; Heller \& Hollabaugh, 1992; Yerushalmi \& Magen, 2006); the creativeness approach in problem solving (Johnstone \& Otis, 2006; Walsh et al., 2007; Cooper et al., 2008; Bennett, 2008); the epistemic games (Tuminaro \& Redish, 2007).

The logical solving model used by the University of Minnesota consisted of similar problem solving strategies: Focus the Problem, which involves determining the question and sketching a picture, and selecting a qualitative approach. The next step, Describe the Physics, includes drawing a diagram, defining symbols, and stating quantitative relationships. The Plan a Solution entails choosing a relationship that includes the target quantity, undergoing a cycle of choosing another relationship to eliminate unknowns and substituting to solve for the target. The step Execute the Plan involves simplifying an expression, and putting in numerical values for quantities if requested. The final step is Evaluate the Answer, which means evaluating the solution for reasonableness, and to check that it is properly stated (Heller \& Heller, 1995).

Bagno and Eylon's (1997) didactic approach constructed through active problem solving by students. According to them, the learning sequence consists of several stages. The first stage is Solve. The student solves a problem in which the relevant relationship between A and B plays a central role. The second stage is Reflect. The student identifies the relationship, compares it to other relevant relationships recognizes differences and likenesses and finally formulated the relationship verbally, symbolically, and visually. The third stage is Conceptualize. The student develops and elaborates the concepts. This is the stage in which common misconceptions are explained and important differences between concepts. The fourth is Apply. The final stage is Link, which the written materials provide compact tables to facilitate retention and retrieval.

In the research of Bolton and Ross (1997), a computer-assisted problem-solving protocol was introduced to long-distance study university students in a simple model of preparation-working-checking. A study by Yerushalmi and Magen (2006) intended to teach students to solve physics problem by transforming context-rich problems into context-poor problems. In this method students are organizing the unknowns and unnecessary contents.

Another method is (Savage \& Williams, 1990) physics-problem solving using realworld problem modeling. This method is purposely designed to solve algebraic mechanics (kinematics and dynamics) problems at university level. The main processes in this method are preparing the model, analyzing the problem, interpreting and confirming the mathematical answer to produce a solution.

Recently, Loucks (2007) introduced a method for solving university physics problems, particularly when algebra is involved, which is similar to Savage and Williams (1990)' problem solving. For Loucks, the most important factor is to set up the problem, so that the solver can determine which equations are suitable. Once it is set up, the problem becomes simply a mathematical problem. Loucks recommended five steps to effectively solve physics problems with algebra; a) identify the type of problem (for example, concept, keyword, feature), b) sort by interval and/or object (e.g., list everything, draw diagram), c) find the equation and unknowns, try to relate the intervals, d) outline solution or make a chain of reaction, e) do the mathematics. 
Mayer (2008) asserted that effective practice in problem solving should be given in a structured way, but not in a step-by-step procedure. He concluded that problem solving programs are most effective when they focus on problem solving not as a single intellectual ability but as a collection of smaller component skills. He stressed that successful problem solving training involves: specific problem solving skills, contextualized tasks that students are expected to perform in school, practice in the process of problem solving, discussion of the problem solving process, teaching problem-solving before students have fully mastered content, knowledge of a domain. He also stressed that problem solving training should be provided in addition to developing domain-specific content knowledge. Students need to learn domain-specific problem solving skills in order to become successful learners in physics.

Consequently, Kowalski et al. (2009) examined on the review of problem solving strategies in computer-based course. Their study was a modification of well-established steps used to teach increased competency in problem solving strategies in engineering courses. They combined problem solving strategy steps with three steps (identifying of the fundamental principle, solving, and checking).

\section{Metacognition in Problem Solving}

Metacognition is defined as knowledge of cognition and regulation of cognition in Flavell's (1976) and Brown's (1978) influential work on metacognition. The term metacognition refers to a students ' knowledge about his/her processes of cognition and the ability to control and monitor those processes as a function of the feedback received via outcomes of learning. Thus, two essential components comprise metacognition: knowledge and control. Metacognition knowledge refers to what a student understands and believes about a subject matter or a task, and the judgments s/he makes in allocating cognitive resources as a result of that knowledge. Metacognition control refers to the approaches and strategies a student devises to achieve specific learning goals and the degree to which the learner organizes, monitors, and modifies those operations to ensure effective learning (Flavel, 1979; Hollingworth \& McLoughlin, 2001; Metallidou, 2009).

Metacognitive knowledge consists of declaration, procedure, and strategy. There is a general agreement among researchers that declarative knowledge (facts and concepts) alone is not sufficient for better performance and achievement. It must be followed by procedural (how to use these facts and concepts in methods or procedures) and strategic knowledge (knowledge needed to organize the process of solving new problems) knowledge. The latter are referring to the selection of the appropriate strategy for different kind of problems and the evaluation of the effectiveness of each strategy (Metallidou, 2009).

With regard to metacognitive control, attention resources, existing cognitive strategies, and awareness of breakdowns in comprehension are all enhanced by metacognitive knowledge and skills. Thus, metacognition is important to regulate and improve their cognitive tactics and strategies used in problem solving process (Hollingworth \& McLoughlin, 2001; Desoete, 2008).

It has been observed that during problem solving process students having metacognitive behaviors are more controlled; they try to break the complex problems into sample parts and ask questions themselves for clarifying their thoughts. Schoenfeld (1985) states that when one encounter with failures in problem solving techniques, control skills (metacognition) will be helpful for applying strategies successfully.

Most of the research on metacognition in the open literature has been applied on the primary and secondary school students. The studies at the university level generally have covered the mathematical topic. Thus, the attention is directed to mathematics-problem 
solving which shares some likeness with physics in terms of the nature of the subject (Stillman \& Galbraith, 1998; Goos et al., 2002; Kramarski et al., 2002; Georghiades, 2004; Schraw et al., 2006). Therefore, the methods used by some researchers in observing physics problem solving and metacognitive skills in their studies were summarized. From the summary of science problem solving research by Garrett (1986), there have been four methods used to examine physics problem solving between the 1950s and 1980s. These are experimental/statistical research (four studies), case study (one study), individual interview (one study), and protocol analysis (seven studies). With the exception of one study that used statistical measurement (Bascones et al., 1985) and two that used interviews (Henderson et al., 2001; Kuo, 2004), most of the studies produced thinking aloud protocols (Simon \& Simon, 1978; Larkin \& Reif, 1979; Larkin et al., 1980; Chi et al., 1981; Larkin, 1981; Amigues, 1988; Robertson, 1990). Also it was reported that those metacognitive behaviours improved with thinking-aloud protocols which could also be a suitable method for physics problemsolving (Anderson \& Nashon, 2006; Meijer et al., 2006).

Probably Meijer et al. (2006) is the only published empirical paper in research mentioned above. The purpose of their study was to build taxonomy of metacognitive activities in text-reading and problem solving. History text-reading and physics problem solving were chosen to discount the issue of domain-specific, so both natural science and humanity subjects were considered. Sixteen students in Netherlands completed four assignments that followed a physics text involving "motion" using thinking-aloud so thinking-aloud protocols could be generated and analysed. As a result, six main metacognitive activities were identified: orientating, planning, executing, monitoring, evaluation, and elaboration. Unfortunately, a detailed taxonomy could not be produced from this extensive research because the researchers restrained themselves in a too objective paradigm. The use of statistical correlation and the high expectation of reliability in the researchers' coding of the thinking-aloud protocol forced the researchers to reduce the level of detail of the taxonomy.

Amigues (1988) and Anderson \& Nashon (2006) reported studies in group problemsolving at secondary school level. The latter conducted an interpretive study in examining the influence of metacognition in knowledge construction through physics problem solving (kinematics) at an amusement park among years 11 and 12 students (aged 16-18) in Canada. Through protocols analysis of the conversation between three students in a group, it was discovered that the student with higher metacognitive profile (i.e., control, monitoring, awareness, evaluation, planning, and self-efficacy) can easily influence and change the knowledge construct of other students with lower metacognitive profile (especially in metacognitive awareness) even if the knowledge construct of the student with high metacognitive profile was wrong.

Studies on metacognition have proven that there is a strong correlation between problem solving and metacognition. The students with a higher level of metacognitive skills become successful in problem solving (Schoenfeld, 1985; Lucangeli et al., 1995). Kapa (2007) examined the effect of various types of training in metacognitive support mechanism on the students performance on structured (near transfer) and open-ended (far transfer) problems in a computerized metacognitive environment. Different experimental groups received training either during problem solving phases or only after the conclusion of problem solving process. Metacognitive training on both the process and the product phases significantly affected performance on near and far transfer problems in the experimental groups as compared to the control group. There exist positive and meaningful increases in the achievement of students using instruction activities towards developing metacognitive skills (Kramarski et al., 2002; Teong, 2002). 
Goos et al. (2002) stated that problem solving process requires analyzing the given information about the problem, organizing the information, preparing an action plan and assessing all the operations carried out. These operations of problem solving require one to arrange each level and step and decide at the same time. And all these operations performed during the process are skills which constitute the character of metacognition. However, Hollingworth and McLoughlin (2001) claimed that problem solving operations such as definition of problem, practice, and controlling the outcome are not enough for learning. It is not sufficient to know what to do. It is necessary to know when to apply similar strategies, too. According to Montague (1992), three most commonly used metacognitive skills during problem solving should be self-instruction, self-questioning, and self-monitoring. Selfinstruction helps children to determine and manage previously used problem solving strategies while working on a problem. Through the introduction of internal dialogues, selfquestioning enables them to systematically analyze the given information about the problem and manage appropriate cognitive skills. Self-monitoring allows students to monitor their own general performances during problem solving operations and be sure about the appropriateness of the method they use (Ozsoy \& Ataman, 2009; Artz \& Armour-Thomas, 1992).

Considering the vagueness of the definition and theory of metacognition, more difficulty is created in measuring metacognition - an inner behaviour like cognition which used to be perceived as impossible to observe by behaviourists (Mayer, 1991). According to Tobias and Everson (2000), metacognition is usually assessed in two principal ways: observations of students' performance or by self-report inventories. There are a few popular techniques used in measuring metacognitive knowledge and processes: self-report, error detection, interview (structured, semi structured, unstructured, open-ended, closed, introspective, and retrospective) and thinking-aloud (Pintrich et al., 2000; Baker \& Cerro, 2000). There is a large body of experimental research in metacognition which usually picks on specific attributes to be examined, especially those parts which are easier to measure (Schraw et al., 2006).

\section{Discussion}

Problem solving strategy steps are important for students to use while solving problems. Usage of these models of problem solving strategy may be enhanced the creativeness and awareness of the students. They may improve the thinking process of students systematically. However, problem solving strategy models have a few drawbacks. It seems that these problem solving strategy steps take students' time, students should spend more time and effort to engage with the problem in both conceptual and problem solving aspects. Another drawback may be the unnecessary problem solving strategy steps usage for basic problems which are plug-chuck type.

With regard to metacognition, different researchers have proposed different characteristics of metacognitive skills in problem solving. The majority of them accepted that planning, monitoring, and evaluating are the metacognitive skills required in problem solving. Metacognition consists of at least metacognitive knowledge and monitoring. What the students know and believe about their thinking, however, varies. One has to distinguish between the students' metacognitive knowledge and their awareness of using it. It is possible for the students to have some metacognitive knowledge about how they think in physics problem solving without using it consciously - or perhaps not consciously using it at all during problem solving. Therefore, students who possess rich metacognitive knowledge do not necessarily perform metacognitive monitoring using the metacognitive knowledge that they have, and hence may not perform well in problem solving. 


\section{Conclusion}

Literature review on problem solving in physics education showed that problem solving strategies (in general; analyze, solve, and check) used by students are very effective and enhance the performance of the students. Unfortunately only teaching these problem solving strategies is not sufficient for helping students to develop true expertise. Also, instructors should use any of these problem solving strategy models in their lectures.

When all of the problem solving strategy models examined in this research are analysed, the fundamental principles of the problem solving may be summarized for the instructors as follows.

I. Identifying the Fundamental Principle(s): In the first and most important step, a student should accurately identify and understand the problem. A student should examine both the qualitative and quantitative aspects of the problem and interpret the problem in light of his/her own knowledge and experience. This enables a student to decide whether information is important and what information may be needed. In this step students must: (i) simplify the problem situation by describing it with a diagram or a sketch in terms of simple physical objects and essential physical quantities; (ii) restate what you want to find by naming specific mathematical quantities; (iii) represent the problem with formal concepts and principles.

II. Solving: A student uses qualitative understanding of the problem to prepare a quantitative solution. Dividing the problem into subproblems is an effective strategy for constructing the solution. Thus, the solution process involves repeated applications of the following two steps: (i) choosing some useful subproblems, (ii) carrying out the solution of these subproblems. These steps can then be recursively repeated until the original problem has been solved. The decisions needed to solve a problem arise from choosing subproblems. The two main obstacles can be: (i) lack of needed information, (ii) available numerical relationships which are potentially useful, but contain undesirable features. These choices are promoted if there are only few reasonable options among which a student needs to choose. An effective organization of knowledge has crucial importance in making easy the decisions needed for problem solving. The organization done after applying the particular principle is facilitated by all of a student's previously gained technical knowledge. The final step contains plugging in all the relative quantities into the algebraic solution to determine a numerical value for the wanted unknown quantity (ies).

III. Checking: In the final step, a student should check the solution to assess whether it is correct and satisfactory and to revise it properly if any shortages are detected by following this checklist; (i) Has all wanted information been found? (ii)Are answers expressed in terms of known quantities? (iii)Are units, signs or directions in equations consistent? (iv) Are both magnitudes and directions of vectors specified? (v) Are answers consistent with special cases or with expected functional dependence? (vi) Are answers consistent with those obtained by another solution method? (vii) Are answers and solution as clear and simple as possible? (viii) Are answers in general algebraic form?

Metacognition has been suggested as an important factor in physics problem solving recently, the metacognitive aspect of problem solving in order to better understand the students' thinking processes in physics problem solving. Some metacognitive skills- planning, monitoring, and evaluating- should be incorporated into the problem solving instruction to further refine students` problem solving skill. Based on the research conducted in the literature, instead of just telling problem-solving strategies, the instructional design should be modified to promote cognitive process that build structured knowledge and develop desirable habits of mind, and to guide students through the stages of cognitive development. 


\section{References}

Amigues, R. (1988). Peer interaction in solving physics problems: Sociocognitive confrontation and metacognitive aspects. Journal of Experimental Child Psychology,45, 141-158.

Anderson, D., Nashon, M. (2006). Predators of knowledge construction: Interpreting students' metacognition in an amusement park physics program. Science Education,1-23.

Artz, F., Armour-Thomas, E. (1992). Development of a cognitive-metacognitive framework for protocol analysis of mathematical problem solving in small groups. Cognition and Instruction, 9, 137-175.

Bagno, E., Eylon, Bat-S. (1997). From problem solving to a knowledge structure: An example from the domain of electromagnetism. American Journal of Physics, 65, 726-736.

Baker, L. Cerro, L. C. (2000). Assessing metacognition in children and adults. In G. Schraw \& J. C. Impara (Eds.), Issues in the measurement of metacognition (pp.99- 145). Lincoln: Buros Institute of Mental Measurements.

Bascones, J., Novak, V., Novak, J. D. (1985). Alternative instructional systems and the development of problem-solving skills in physics. European Journal of Science Education, 7(3), 253-261.

Bennett, W. (2008). Problem solving: can anybody do it? Chemistry Education Research and Practice, 9, 60-64.

Bolton. J., Ross, S. (1997). Developing students’ physics problem-solving skills. Physics Education, 32, 176-185.

Brown, A. L. (1978). Knowing when, where, and how to remember: A problem of metacognition. In R. Glaser (Eds.), Advances in instructional psychology (Vol. 1) (pp.77-165). New Jersey: Lawrence Erlbaum Associates.

Chi, T., Feltovich, P., Glaser, R. (1981). Categorization and representation of physics problems by experts and novices. Cognitive Science, 5, 121-152.

Cooper, M., Cox, C., Nammouz, M., Case, E. (2008). An assessment of the effect of collaborative groups on students' problem-solving strategies and abilities. Journal of Chemical Education, 85, 866-872.

Desoete, A. (2008). Multi-method assessment of metacognitive skills in elementary school children: How you test is what you get. Metacognition and Learning, DOI.10.1007/s11409-008-9026-0.

Dewey, J. (1910). How we think. London: D. C. Heath \& Company.

Dufrense, R., Gerace, W., Leonard, J. (1997). Solving physics problems with multiple representations. Physics Teacher, 35, 270.

Elshout, J. J. (1987). Problem-solving and education. In E. De Corte, H. Lodewijks, R.Parmentier \& P. Span (Eds.), Learning and instruction: European research in an international context (Vol. 1) (pp.259-274). Oxford: Leuven University Press and Pergamon Press.

Flavell, J. (1976). Metacognitive aspects of problem solving. In L.B. Resnick (Eds.), The nature of intelligence (pp.231-235). New Jersey: Lawrence Erlbaum Associates. 
Flavell, J. (1979). Metacognitive and cognitive monitoring: A new area of cognitive developmental inquiry. American Psychologist, 34, 906-911.

Garrett, R. M. (1986). Problem-solving in science education. Studies in Science Education, 13, 70-95.

Georghiades, P. (2004). From the general to the situated: Three decades of metacognition. International Journal of Science Education, 26(3), 365-383.

Gil-Perez, D., Dumas-Carre, Caillot, M., \& Martinez-Torregrosa, J. (1990). Paper and pencil problem solving in the physical sciences as a research activity. Studies in Science Education, 18, 137-151.

Goos, M., Galbraith, P., Renshaw, P. (2002). Socially mediated metacognition: Creating collaborative zones of proximal development in small group problem solving. Educational Studies in Mathematics, 49, 193-223.

Harskamp, E., Ding, N. (2006). Structured collaboration versus individual learning in solving physics problems. International Journal of Science Education, 14, 1669-1688.

Heller, I., Reif, F. (1984). Prescribing effective human problem-solving processes: Problem description in physics. Cognition and Instruction, 1, 77-216.

Heller, P., Keith, R., Anderson, S. (1992). Teaching problem solving through cooperative grouping. Part 1: Group versus individual problem solving. American Journal of Physics, 60, 627-636.

Heller, P., Hollabaugh, M. (1992). Teaching problem solving through cooperative grouping. Part 2: Designing problems and structuring groups. American Journal of Physics, 60, 637-644.

Heller, K., Heller, P. (1995). The competent problem solver, a strategy for solving problems in physics, calculus version (2nd ed.). Minneapolis, MN: McGraw-Hill.

Henderson, C., Heller, K., Heller, P., Kuo, V. H. Yerushalmi, E. (2001). Instructors' ideas about problem solving - Setting goals. Proceedings of Physics Education Research Conference, Rochester, New York, July 2001.

Hollingworth, W., McLoughlin, C. (2001). Developing science students' metacognitive problem solving skills on-line. Australian J. of Educational Technology, 17, 50-63.

Johnstone, H., Otis, H. (2006). Concept mapping in problem based learning: a cautionary tale. Chemistry Education Research and Practice, 7, 84-95.

Kapa, E. (2007). Transfer from structured to open-ended problem solving in a computerized metacognitive environment. Learning and Instruction, 17, 688-707.

Kneeland, S. (1999). Effective problem solving: Hoe to understand the process and practice it successfully. How to Books.

Kohl, P., Rosengrant, D., Finkelstein, N. (2007). Strongly and weakly approaches to teaching multiple representation use in physics. Physical Review Special Topics-Physics Education Research, 3, 1-10.

Kohl. P., Finkelstein, N. (2008). Patterns of multiple representation use by experts and novices during physics problem solving. Physical Review Special Topics-Physics Education Research, 010111, 1-13. 
Kowalski, F., Gök, T., Kowalski, S. (2009). Using Tablet PCs to strengthen problem-solving skills in an upper-level engineering physics course, 39th ASEE/IEEE Frontiers in Education Conference, October 18- 21, 2009, San Antonio, TX

Kramarski, B., Mevarech, R., Arami, M. (2002). The effects of metacognitive instruction on solving mathematical authentic tasks. Educational Studies in Mathematics, 49, 225-250.

Kuo, V. (2004). An explanatory model of physics faculty conceptions about the problem solving process. Unpublished doctoral thesis, University of Minnesota.

Larkin, H. (1979). Processing information for effective problem solving. Engineering Education, 70, 285-288.

Larkin, H., Reif, F. (1979). Understanding and teaching problem-solving in physics. European Journal of Science Education, 1, 191-203.

Larkin, H., McDermott, J., Simon, P., \& Simon, A. (1980). Model of competence in solving physics problems. Cognitive Science, 4, 317-345.

Larkin, J. H. (1981). Enriching formal knowledge: A model for learning to solve textbook physics problems. In J. R. Anderson (Eds.), Cognitive skills and their acquisition (pp.311- 334). New Jersey: Lawrence Erlbaum Associates.

Loucks, S. E. (2007). Introductory physics with algebra: Mastering problem-solving. US: John Wiley \& Sons.

Lucangeli, D., Galderisi, D., Cornoldi, C. (1995). Specific and general transfer effects of meta-memory training. Learning Disabilities Research and Practice, 10, 11-21.

Mayer, R. E. (1991). Thinking, problem solving, cognition (2nd ed.). New York: W. H. Freeman and Company.

Mayer, R. E. (2008). Learning and Instruction. Upper Saddle River, NJ: Prentice Hall.

Mazur, E. (1997). Peer Instruction: A user's manual. Upper Saddle River, NJ: Prentice Hall.

McDermott, C. (1991). Millikan Lecture 1990: What we teach and what is learned-closing the gap. American Journal of Physics, 59, 301-315.

Meijer, J., Veenman, J., van Hout-Wolters, B. (2006). Metacognitive activities in textstudying and problem- solving: Development of a taxonomy. Educational Research and Evaluation, 12, 209-237.

Mestre, J. P. (2001). Implication of research on learning. Physics Education, 36, 44-51.

Metallidou, P. (2009). Pre-service and in-service teachers' metacognitive knowledge about problem-solving strategies. Teaching and Teacher Education, 25, 76-82.

Montague, M. (1992). The effects of cognitive and metacognitive strategy instruction on the mathematical problem solving of middle school students with learning disabilities. J. of Learning Disabilities, 25, 230-248.

Newell, A., Simon, H. A. (1972). Human problem solving. New Jersey: Prentice Hall.

Osborne, J., Dillon, J. (2008). Science education in Europe: Critical reflections. London: Nuffield Foundation.

Ozsoy, G., Ataman, A. (2009). The effect of metacognitive strategy training on mathematical problem solving achievement. International Electronic Journal of Elementary Education, 1, 67-82. 
Pintrich, P. R., Wolters, C. A. Baxter, G. P. (2000). Assessing metacognition and selfregulated learning. In G. Schraw, \& J. C. Impara (Eds.), Issues in the measurement of metacognition (pp.43-97). Lincoln: Buros Institute of Mental Measurements.

Pol, H. (2005). Solving physics problems with the help of computer-assisted instruction. International J. of Sci. Education, 27, 451-469.

Polya, G. (1945). How to solve it. New Jersey: Princeton University Press.

Reif, F., Larkin, H., Brackett, C. (1976). Teaching general learning and problem-solving skills. American Journal of Physics, 44, 212-217.

Reif, F. (1981). Teaching problem solving: A scientific approach. The physics Teacher, 19, 310-316.

Reif, F., Heller, I. (1982). Knowledge structures and problem solving in physics. Educational Psychologist, 17, 102-127.

Reif, F. (1995). Millikan Lecture 1994: Understanding and teaching important scientific thought process. American Journal of Physics, 59, 891.

Robertson, W. C (1990). Detection of cognitive structure with protocol data: Redicting performance on physics transfer problems. Cognitive Science, 14, 253-280.

Savage, M., Williams, J. (1990). Mechanics in action-modelling and practical investigations. Cambridge: Cambridge University Press.

Schraw, G, Crippen, K. J. \& Hartley, K. (2006). Promoting self-regulation in science education: Metacognition as part of a broader perspective on learning. Research in Science Education, 36, 111-139.

Schunk, D. H. (2000). Learning theories - An educational perspective. New Jersey: Prentice Hall.

Schoenfeld, H. (1985). Mathematical problem solving. San Diego: Academic Press.

Simon, D. P. \& Simon, H. A. (1978). Individual differences in solving physics problems. In R. S. Siegler (Eds.), Children thinking: What develop? (pp.325-348). New Jersey: Lawrence Erlbaum Associates.

Stillman, A., \& Galbraith, L. (1998). Applying mathematics with real world connections: Metacognitive characteristics of secondary students. Educational Studies in Mathematics, 36, 157-195.

Sweller, J. (1988). Cognitive load during problem solving: Effects on learning. Cognitive Science12, 257-285.

Teong, K. (2002). The effect of metacognitive training on mathematical word-problem solving. Journal of Computer Assisted Learning, 19, 46-45.

Tobias, S. \& Everson, H. (2000). Assessing metacognitive knowledge monitoring. In G. Schraw \& J. C. Impara (Eds.), Issues in the measurement of metacognition (pp.141222). Lincoln: Buros Institute of Mental Measurements.

Tuminaro, J., \& Redish, F. (2007). Elements of a cognitive model of physics problemsolving: Epistemic games. Physical Review Special Topics-Physics Education Research, 3, 1-22.

Van-Heuvelen, A. (1991). Learning to think like a physicist: A review of research-based instructional strategies. American Journal of Physics, 59, 891-897. 
Walsh, N., Robert, H., \& Bowe, B. (2007). Phenomenographic study of students' problem solving approaches in, physics. Physical Review Special Topics-Physics Education Research, 3, 1-12.

Yerushalmi, E., \& Magen, E. (2006). Same old problem, new name? Alerting students to the nature of the problem-solving process. Physics Education, 41, 161-167. 